\title{
Idiopathic basal ganglia calcification associated with cerebral micro-infarcts: a case report
}

\author{
Takuma Nishimoto* (D), Fumiaki Oka, Hideyuki Ishihara, Mizuya Shinoyama and Michiyasu Suzuki
}

\begin{abstract}
Background: Idiopathic basal ganglia calcification (IBGC) is a rare neurodegenerative disorder characterized by symmetric intracranial calcium deposition. We report a patient with IBGC associated with cerebral infarction due to impairment of cerebrovascular reactivity based on single-photon emission computed tomography (SPECT) with acetazolamide challenge.

Case presentation: A 66-year-old male presented with right conjugate deviation, right hemiparesis and total aphasia due to a convulsive seizure. Brain computed tomography showed symmetric calcifications in the bilateral basal ganglia, thalamus, cerebellar dentate nuclei, which were consistent with IBGC. Diffusion-weighted brain magnetic resonance imaging showed multiple small infarctions in the bilateral cerebral subcortical area. In the search for the cause of cerebral infarction, SPECT with acetazolamide challenge revealed heterogeneous impairment of cerebrovascular reactivity in the whole brain, despite the absence of evidence for steno-occlusive changes in proximal arteries.

Conclusion: Cerebrovascular insufficiency due to the lack of elasticity caused by microvascular calcification might have been one of the pathophysiological features of IBGC in this case. Thus, vascular calcification may cause cerebrovascular disturbance and could lead to ischemic stroke in patients with IBGC.
\end{abstract}

Keywords: Idiopathic basal ganglia calcification, Cerebral micro-infarcts, Vascular calcification, Single photon emission computed tomography, Cerebrovascular reactivity

\section{Background}

Idiopathic basal ganglia calcification (IBGC) was first described by Karl Theodor Fahr in 1930 [1] and is also known as Fahr's disease. IBGC is a rare neurodegenerative disorder characterized by symmetric intracranial calcium deposition mainly in the bilateral basal ganglia and dentate nuclei of the cerebellum in the absence of other causes of secondary calcification. Pathologically, calcium and other mineral deposits are found in the walls of the arterioles, capillaries, small veins and the perivascular space [2,3]. Patients generally present with chronic and progressive symptoms, such as headache, psychiatric symptoms, epilepsy, dementia and Parkinsonism, and there has been one case report of a patient with IBGC who developed ischemic stroke [4]. Herein, we report a case of IBGC in which multiple small

\footnotetext{
* Correspondence: ntakuma@yamaguchi-u.ac.jp

Department of Neurosurgery, Yamaguchi University School of Medicine,

1-1-1, Minamikogushi, Ube, Yamaguchi 755-8505, Japan
}

cerebral infarctions developed after general convulsion, and we discuss the possible mechanisms of ischemic stroke in patients with IBGC.

\section{Case presentation}

A 66-year-old male developed general epileptic convulsion and was admitted to our department one hour after onset. His history included epilepsy and hypertension. On admission, blood pressure was 126/ $84 \mathrm{mmHg}$ and pulse was 82 beats/min with sinus rhythm. Neurological findings included deterioration of consciousness, right conjugate deviation, total aphasia and right hemiparesis with a manual muscle test score of 1/5. The National Institutes of Health Stroke Scale score was 20 points.

Brain computed tomography (CT) showed expansive and symmetric high-density lesions involving the bilateral basal ganglia, thalami and cerebellar dentate nuclei (Fig. 1). Diffusion-weighted brain magnetic resonance imaging (MRI) showed multiple hyperintense

(C) The Author(s). 2018 Open Access This article is distributed under the terms of the Creative Commons Attribution 4.0 International License (http://creativecommons.org/licenses/by/4.0/), which permits unrestricted use, distribution, and 

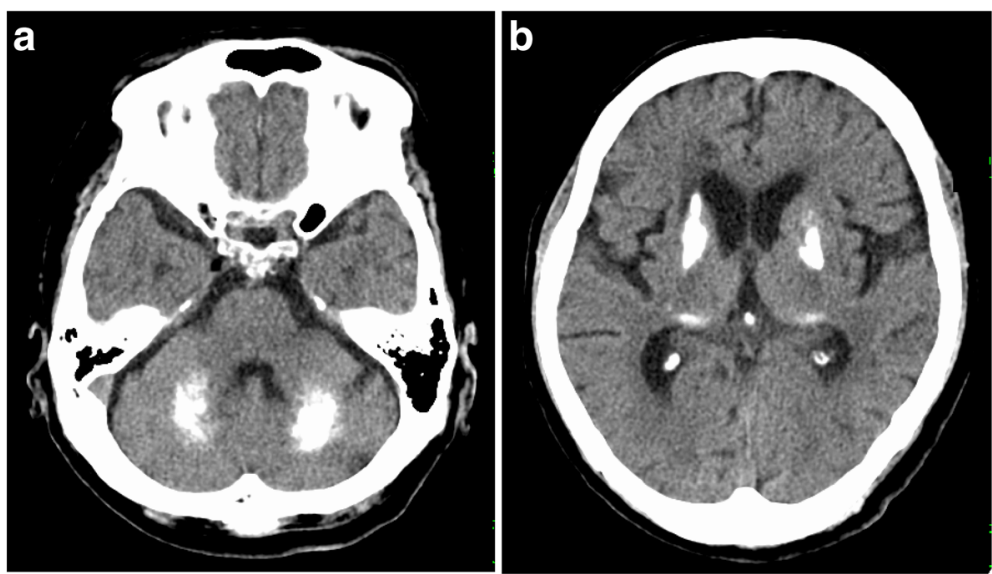

Fig. 1 Brain computed tomography (CT). Axial noncontrast CT showing expansive and symmetric high-density lesions involving the cerebellar dentate nuclei (a) and bilateral basal ganglia and thalami (b)

spots in the bilateral cerebral subcortical area (Fig. 2a, b), which were indicative of acute infarctions. Magnetic resonance angiography did not show any intra- or extracranial arterial occlusion, stenosis or other vascular abnormality (Fig. 2c). MRI using susceptibility-weighted imaging (SWI) showed hypointense lesions coinciding with high-density lesions in CT imaging and multiple hypointense spots in the cerebral parenchyma (Fig. 3a, b). Hypointense lesions that coincided with the highdensity lesions on $\mathrm{CT}$ were detected on both minimal intensity projection and phase images on SWI. Moreover, multiple hypointense spots were found in the bilateral cerebral subcortical white matter, which suggested multiple calcifications in the subcortical area.

After admission, we started administration of an anti-epileptic drug and convulsion was stopped. Thereafter, the patient gradually became alert and fully recovered from the neurological deficits within $24 \mathrm{~h}$ after admission. One day after admission, electroencephalography showed no apparent epileptic discharge or other abnormal findings. Blood pressure was stable all day at 110 to $130 \mathrm{mmHg}$ (systolic) and 60 to $80 \mathrm{mmHg}$ (diastolic).

Examinations were performed to investigate the cause of intracranial calcification. The serum levels of sodium, potassium, chloride, calcium, albumin, phosphorus, intact $\mathrm{PTH}$, thyroid profile, lipid profile and uric acid were all within normal limits, except for lowdensity lipoprotein cholesterol $(133 \mathrm{mg} / \mathrm{dL})$. Complete cell count, C-reactive protein and erythrocyte sedimentation rate were also normal. Autoimmune tests including antinuclear antibody, anti-single and -double-stranded DNA IgG antibody, rheumatoid factor, cytoplasmic and myeloperoxidase antineutrophil cytoplasmic antibody, and anticardiolipin antibody were all negative or within normal limits. To exclude infection, we examined hepatitis $\mathrm{B}$ and $\mathrm{C}$ antibodies and human immunodeficiency virus -1 and -2 antibodies, and performed a venereal disease test, and all were negative. Laboratory tests and whole body CT
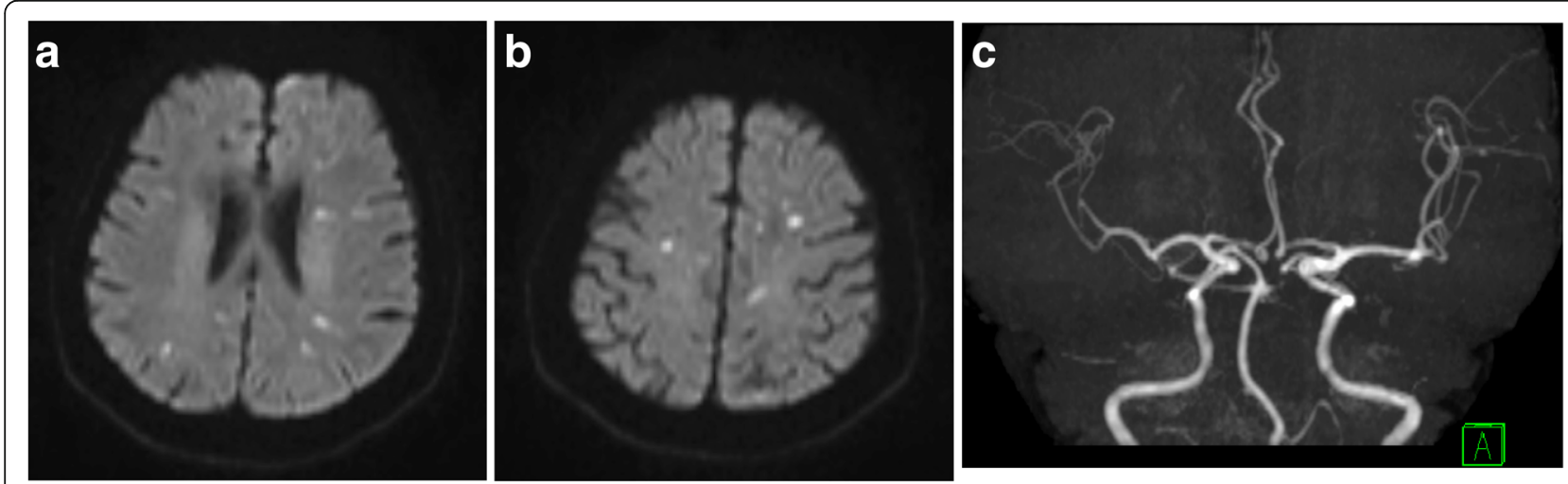

Fig. 2 Brain magnetic resonance imaging (MRI). Axial diffusion-weighted brain MRI showing multiple hyperintense spots in the bilateral cerebral subcortical white matter $(\mathbf{a}, \mathbf{b})$. No intracranial arterial occlusion or stenosis was detected by magnetic resonance angiography (c) 

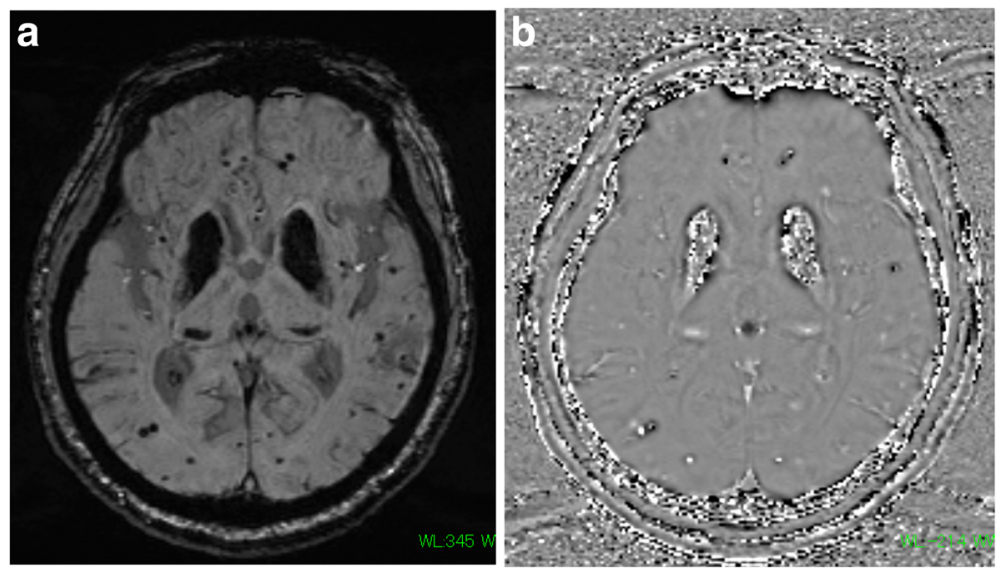

Fig. 3 Brain magnetic resonance imaging (MRI). Minimal intensity projection (a) and phase images (b) on SWI showing multiple hypointense spots in the cerebral sulcus and hypointense lesions coinciding with high-density lesions on CT

showed no evidence of secondary calcification diseases, such as parathyroid disorder, pseudohypoparathyroidism, thyroid disease, other endocrine disease, metabolic disease, collagen diseases, vasculitis, infections (syphilis, hepatitis and HIV) or cysticercosis. Based on these findings, the patient was diagnosed with IBGC.

Concurrently, we performed examinations to investigate the cause of cerebral infarction. Transthoracic echocardiography showed no particular findings. Transesophageal echocardiography revealed a patent foramen ovale; however, there was no evidence of deep vein thrombosis in the lower limbs on contrast CT and ultrasound sonography including Doppler. A 24-h Holter electrocardiogram and monitoring of a continuous 3-lead cable bedside electrocardiogram for 7 days did not show atrial fibrillation or other arrhythmia. Digital subtraction angiography showed no atherosclerotic changes in the proximal arteries.

Quantitative resting cerebral blood flow $(\mathrm{CBF})$ and cerebrovascular reactivity (CVR) were measured by singlephoton emission computed tomography (SPECT) in one session using the dual-table autoradiographic method and dual administration of $\mathrm{N}$-isopropyl-p-[I-123] iodoamphetamine $\left({ }^{123} \mathrm{I}\right.$-IMP), as described in detail elsewhere [5]. For SPECT with drug challenge, acetazolamide $(17 \mathrm{mg} / \mathrm{kg})$ was administered intravenously. Tomographic images were reconstructed and CBF quantification was performed using the QSPECT image reconstruction package [6]. After image reconstruction, images were processed using NEURO FLEXER. This program automatically measures $\mathrm{CBF}$ values at rest and after acetazolamide challenge, and can also calculate CVR, as follows: $C V R=100 \times($ Diamox CBF- resting CBF) / resting CBF [7]. Surprisingly, resting CBF was decreased slightly, and moreover, CVR was severely impaired heterogeneously at basal ganglia or thalami, which were well-calcified areas, and at all other cortical and subcortical areas of the bilateral cerebral hemispheres, in the absence of steno-occlusive changes in the proximal arteries (Fig. 4).

Thorough blood examinations were also conducted to investigate the possibility of coagulation disorders, such as antiphospholipid antibody syndrome, collagen disease, Protein $\mathrm{S}$ and $\mathrm{C}$ deficiency, antithrombin III deficiency and tumor markers, but the results were unremarkable. Finally, even though we could not determine the exact cause of cerebral infarction, we concluded that the lack of vascular reactivity might have played a role in causing multiple cerebral infarctions after convulsive seizure in this patient. To prevent secondary attack, the patient was treated with an antiplatelet drug, cilostazol $(200 \mathrm{mg} /$ day), and was finally discharged from hospital with no neurological deficits.

\section{Conclusions}

IBGC is a rare neurodegenerative disorder characterized by symmetric intracranial calcium deposition. The common sites of calcification in IBGC are the globus pallidus, putamen, caudate nucleus, internal capsule, dentate nucleus, thalamus, and subcortical white matter [8]. CT and MRI using SWI are useful modalities for detecting calcification in IBGC [9-11]. Sahin et al. reported that SWI phase images, in particular, are comparable to CT for identifying intracranial calcification [12]. In our case, both CT and SWI revealed calcium deposits in the bilateral basal ganglia, thalamus and cerebellar dentate nuclei. Furthermore, SWI showed multiple hypointense spots in the subcortical white matter, which indicated calcium deposition in areas that could not be detected by CT.

Only one case report has shown a clinical relationship between cerebral infarction and IBGC [4]. This report showed young onset of ischemic stroke at the internal capsule, similar to well-known lacunar infarction, in a 

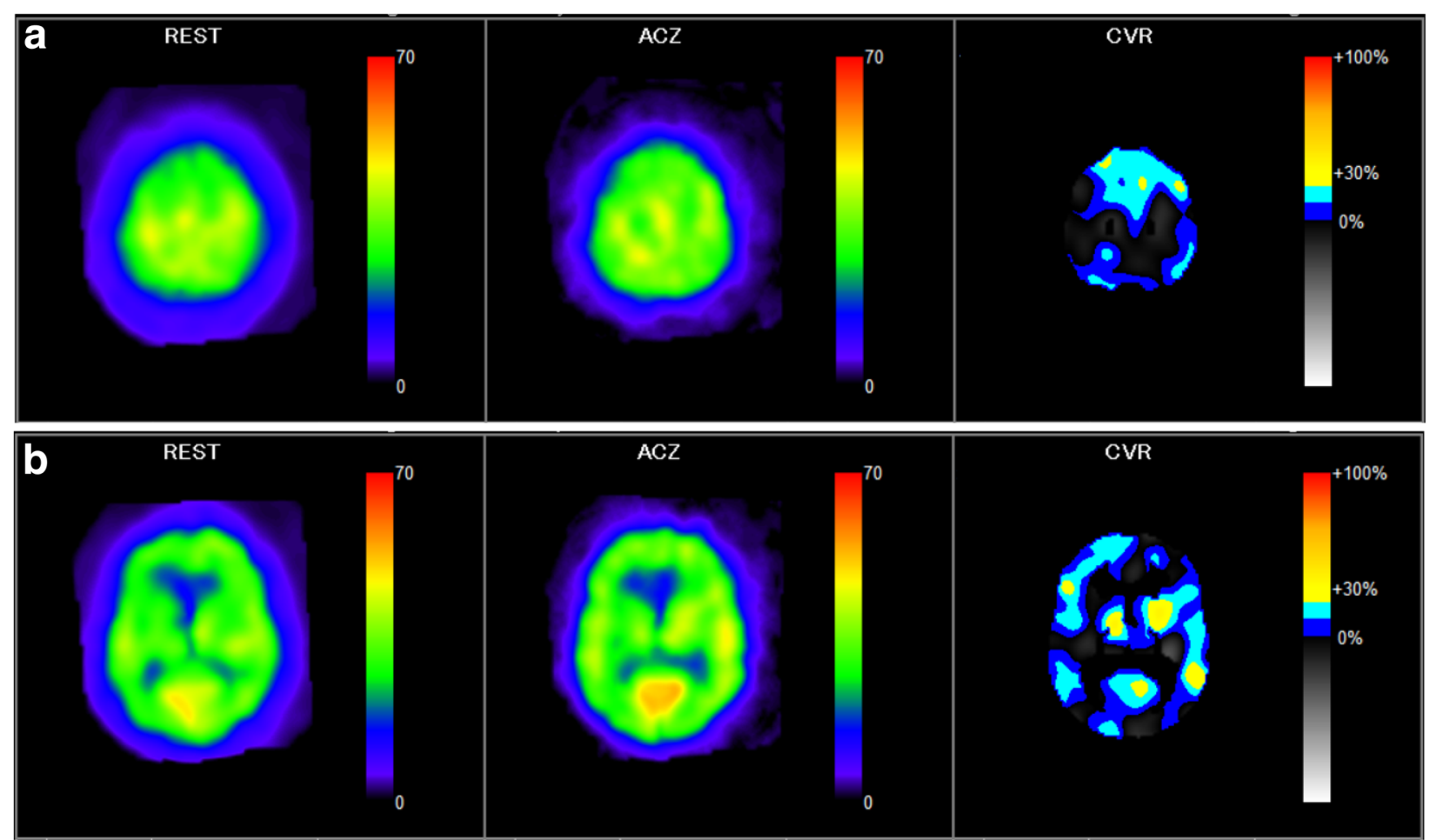

Fig. 4 Single-photon emission computed tomography (SPECT). Quantitative CBF assessment with SPECT showed a mild decrease in resting CBF and a severe decrease in CVR over almost all the cerebral area in an acetazolamide-tolerance test. Images are shown at the level of (a) the centrum semiovale and (b) the basal ganglia. ACZ; acetazolamide, CVR; cerebrovascular reactivity

patient with a IBGC. In contrast, in our case, cerebral infarctions occurred simultaneously in multiple subcortical areas in both hemispheres after a convulsive seizure. Although the mechanism of infarction is not clear in our case, it might be related to impaired CVR throughout the whole brain, as revealed by SPECT. Impaired CVR is a risk factor for stroke $[13,14]$ and we assume that impaired CVR might have been related to calcification of blood vessels. Vascular calcifications compromise arterial elastance, and calcification in IBGC is mainly observed in the walls of capillary vessels, arterioles, small veins and the perivascular space $[2,3]$. Multiple infarctions may have been another cause of impairment of CVR, since a trend for a negative correlation between CVR and the number of lacunar infarctions has been reported [13]. Additionally, since impairment of CVR in hypertensive patients has been reported, history of hypertension in this patient may also be associated with impaired CVR [15]. Based on the MRI and SPECT findings, we postulate that one of the mechanisms of cerebral infarction in our patient with IBGC was a demand-supply mismatch during convulsion. In other words, the convulsive seizure increased the demand for cerebral blood flow, but the demand could not be met due to the decrease in vascular diastolic capacity related to calcification, and this consequently resulted in cerebral infarction.
This is the first reported case of a patient with IBGC with impairment of CVR found on SPECT with acetazolamide challenge. Vascular calcification may cause cerebrovascular disturbance and could lead to ischemic stroke or other phenotypes, such as progressive cognitive impairment, in patients with IBGC. Further studies in a larger numbers of patients are required to understand the pathophysiology of IBGC.

\section{Abbreviations \\ ${ }^{123}$ I-IMP: N-isopropyl-p-[I-123] iodoamphetamine; CBF: Cerebral blood flow; CT: Computed tomography; CVR: Cerebrovascular reactivity; IBGC: Idiopathic basal ganglia calcification; MRI: Magnetic resonance imaging; SPECT: Single- photon emission computed tomography; SWI: Susceptibility-weighted imaging}

Funding

No financial support was provided for this study.

\section{Availability of data and materials}

All data and material supporting the conclusions of this article is included in the article. Identifying/confidential information has not been and shall not be shared.

\section{Authors' contributions}

$\mathrm{TN}$ and FO examined the patient and drafted the manuscript. TN, FO, HI and MS1 evaluated the neuroimaging findings and gave important clinical opinions. MS2 participated in the design of the case report and helped to draft the manuscript. All authors read and approved the final manuscript.

Ethics approval and consent to participate Not applicable. 


\section{Consent for publication}

Written informed consent was obtained from the patient for publication of this case report and accompanying images. A copy of the written consent is available for review by the Editor-in-Chief.

\section{Competing interests}

The authors declare that they have no competing interests.

\section{Publisher's Note}

Springer Nature remains neutral with regard to jurisdictional claims in published maps and institutional affiliations.

Received: 28 September 2017 Accepted: 13 April 2018

Published online: 17 April 2018

\section{References}

1. Fahrni GS. Pregnancy complicating hyperthyroidism and following thyroidectomy. Can Med Assoc J. 1930;23(5):645-7.

2. Duckett S, Galle P, Escourolle R, Poirier J, Hauw JJ. Presence of zinc, aluminum, magnesium in striopalledodentate (SPD) calcifications (Fahr's disease): electron probe study. Acta Neuropathol. 1977;38(1):7-10.

3. Kimura T, Miura T, Aoki K, Saito S, Hondo H, Konno T, Uchiyama A, Ikeuchi T, Takahashi H, Kakita A. Familial idiopathic basal ganglia calcification: histopathologic features of an autopsied patient with an SLC20A2 mutation. Neuropathology. 2016;36(4):365-71.

4. Yang CS, Lo CP, Wu MC. Ischemic stroke in a young patient with Fahr's disease: a case report. BMC Neurol. 2016;16:33.

5. Oka F, Ishihara H, Kato S, Higashi M, Suzuki M. Cerebral hemodynamic benefits after contralateral carotid artery stenting in patients with internal carotid artery occlusion. AJNR Am J Neuroradiol. 2013;34(3):616-21.

6. Kim KM, Watabe H, Hayashi T, Hayashida K, Katafuchi T, Enomoto N, Ogura T, Shidahara M, Takikawa S, Eberl S, et al. Quantitative mapping of basal and vasareactive cerebral blood flow using split-dose 123liodoamphetamine and single photon emission computed tomography. Neurolmage. 2006;33(4):1126-35.

7. Yamada S, Kobayashi M, Watanabe Y, Miyake H, Oshima M. Quantitative measurement of blood flow volume in the major intracranial arteries by using 123i-iodoamphetamine SPECT. Clin Nucl Med. 2014;39(10):868-73.

8. Lester J, Zuniga C, Diaz S, Rugilo C, Micheli F. Diffuse intracranial calcinosis: Fahr disease. Arch Neurol. 2006;63(12):1806-7.

9. Haacke EM, Cheng NY, House MJ, Liu Q, Neelavalli J, Ogg RJ, Khan A, Ayaz M, Kirsch W, Obenaus A. Imaging iron stores in the brain using magnetic resonance imaging. Magn Reson Imaging. 2005;23(1):1-25.

10. Manyam BV, Bhatt MH, Moore WD, Devleschoward AB, Anderson DR, Calne DB. Bilateral striopallidodentate calcinosis: cerebrospinal fluid, imaging, and electrophysiological studies. Ann Neurol. 1992;31(4):379-84.

11. Tong KA, Ashwal S, Obenaus A, Nickerson JP, Kido D, Haacke EM. Susceptibility-weighted MR imaging: a review of clinical applications in children. AJNR Am J Neuroradiol. 2008;29(1):9-17.

12. Sahin N, Solak A, Genc B, Kulu U. Fahr disease: use of susceptibilityweighted imaging for diagnostic dilemma with magnetic resonance imaging. Quant Imaging Med Surg. 2015;5(4):628-32.

13. Molina C, Sabin JA, Montaner J, Rovira A, Abilleira S, Codina A. Impaired cerebrovascular reactivity as a risk marker for first-ever lacunar infarction: a case-control study. Stroke. 1999;30(11):2296-301.

14. Yonas H, Smith HA, Durham SR, Pentheny SL, Johnson DW. Increased stroke risk predicted by compromised cerebral blood flow reactivity. J Neurosurg. 1993;79(4):483-9.

15. Maeda H, Matsumoto M, Handa N, Hougaku H, Ogawa S, Itoh T, Tsukamoto Y, Kamada T. Reactivity of cerebral blood flow to carbon dioxide in hypertensive patients: evaluation by the transcranial Doppler method. J Hypertens. 1994;12(2):191-7.

\section{Ready to submit your research? Choose BMC and benefit from:}

- fast, convenient online submission

- thorough peer review by experienced researchers in your field

- rapid publication on acceptance

- support for research data, including large and complex data types

- gold Open Access which fosters wider collaboration and increased citations

- maximum visibility for your research: over $100 \mathrm{M}$ website views per year

At BMC, research is always in progress.

Learn more biomedcentral.com/submissions 